Review

\title{
Toward Non-Enzymatic Ultrasensitive Identification of Single Nucleotide Polymorphisms by Optical Methods
}

\section{Kira Astakhova}

Nucleic Acid Center, Department of Physics, Chemistry and Pharmacy, Campusvej 55, Odense M 5230, Denmark; E-Mail: ias@ @sdu.dk; Tel.: +45-6550-25-23; Fax: +45-6615-87-80

Received: 2 February 2014; in revised form: 7 July 2014 / Accepted: 8 July 2014 /

Published: 22 July 2014

\begin{abstract}
Single nucleotide polymorphisms (SNPs) are single nucleotide variations which comprise the most wide spread source of genetic diversity in the genome. Currently, SNPs serve as markers for genetic predispositions, clinically evident disorders and diverse drug responses. Present SNP diagnostics are primarily based on enzymatic reactions in different formats including sequencing, polymerase-chain reaction (PCR) and microarrays. In these assays, the enzymes are applied to address the required sensitivity and specificity when detecting SNP. On the other hand, the development of enzyme-free, simple and robust SNP sensing methods is in a constant focus in research and industry as such assays allow rapid and reproducible SNP diagnostics without the need for expensive equipment and reagents. An ideal method for detection of SNP would entail mixing a DNA or RNA target with a probe to directly obtain a signal. Current assays are still not fulfilling these requirements, although remarkable progress has been achieved in recent years. In this review, current SNP sensing approaches are described with a main focus on recently introduced direct, enzyme-free and ultrasensitive SNP sensing by optical methods.
\end{abstract}

Keywords: SNP; diagnostics; PCR; enzyme-free assays; fluorescence; nanoparticles; locked nucleic acids; polyaromatic hydrocarbons

\section{Introduction}

The decoding of the human genome gave information on more than three million single nucleotide polymorphisms (SNPs) which present important targets to study when addressing the influence of small genetic variations on complex phenotypes [1,2]. Obtaining a deeper understanding of the latter, e.g., on correlation between certain SNPs and disease states and individual pharmacological responses, 
offers the possibility of improved personalized health-care. To realize this task on a worldwide scale, highly sensitive, robust and inexpensive SNP detection assays are in demand (References [1,2], and the following examples of this review).

Numerous strategies have been developed for sensing SNP, and most of the detection methods rely on optical techniques, such as fluorescence, due to their convenience, relatively low cost and robustness. The main challenges of these assays is to gain sufficient sensitivity and specificity to sense one single SNP in 1,000,000,000 bp complex human genome [1,2]. This review describes the most recent developments in both enzyme-based and enzyme-free methods for diagnostics of SNP in research and clinics. The major goal of this review is to underline the progress of SNP diagnostics towards simple, robust and ultrasensitive technologies which will make SNP analysis both accessible and reliable for personalized health care and for future research (Figure 1).

Figure 1. Representative one-step detection of single nucleotide polymorphisms (SNPs) by optical methods. SNP is indicated as a blue cross.

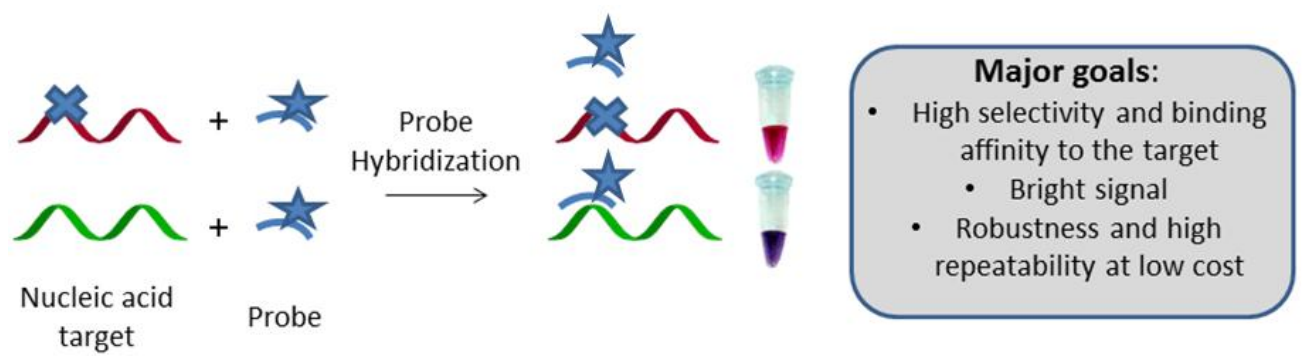

\section{Enzyme-Based Detection of SNP}

Among other methods, the allele-specific polymerase-chain reaction (PCR) allows reduction in the complexity of target DNA by amplification of a specific region [3-6]. In allele-specific PCR, the specificity of oligonucleotides can be dramatically improved using synthetic nucleic acid analogues such as locked nucleic acid (LNA, Chart 1) [7-14]. LNA's unique bicyclic structure allows it to adopt the RNA mimicking N-type furanose conformation. As a result, structural and thermodynamic parameters of LNA/DNA oligonucleotides are changed compared to non-modified DNA (e.g., high binding affinity and mismatch specificity to DNA - especially RNA targets - and resistance to enzymatic degradation). Although optimal positioning of LNA units in PCR primers still needs a systematic evaluation, several allele-specific LNA/DNA primers and probes have been successfully applied and also characterized by thermodynamic and design parameters $[15,16]$. Clearly, further studies aiming at development of general design parameters for LNA/DNA primers and probes will enable enhanced application of LNA/DNA in molecular diagnostics of SNP by PCR.

Amplified DNA targets can be analyzed for the presence of SNP by different direct and indirect hybridization strategies including intercalator dyes in allele-specific digital PCR (dPCR) [17], sequence-specific fluorogenic probes [18], electrochemical sensors [19-24] and diagnostic nano-materials [25]. Electrochemical sensing is a promising approach for SNP detection which takes advantage of synthetic oligonucleotide probes and the large thermodynamic changes in enthalpy and entropy. These thermodynamic changes result from major conformational rearrangements that take place upon binding a perfectly-matched target, resulting in a large-scale change in the faradaic current. 
Being a sensitive technique, electrochemical sensing still requires rather expensive equipment and lacks robustness when compared to optical methods utilizing e.g. nano-particles and fluorescence spectroscopy [17-25]. Next several efficient colorimetric assays for SNP sensing in amplified DNA have been developed based on modified gold nano-particles (Au-nps; Figure 2) [26]. These methods are based on the unique optical properties of colloid gold, whose color is strongly dependent on the degree of aggregation of Au-nps in suspension and on the concentration of electrolytes such as salt [26]. Aggregation of Au-nps can be easily monitored by absorption spectroscopy or by visual observation. Salt-induced aggregation of Au-nps can be prevented by binding negatively charged oligonucleotides which can be a base for a colorimetric assay of DNA. Thus, Au-nps were exposed to the PCR mixture after the reaction containing two additional probes, binding and overlapping the position of the possible SNP (Figure 2A) [26]. Prior to adding Au-nps, the mixture was denatured and annealed below the melting temperature of the complementary probes. If a mutation exists in the target sequence, the PCR product will remain attached to the amplified compliment while the probes will be dehybridized hence, preventing salt-induced aggregation of added Au-nps giving a change of color. By doing this, Li and co-workers could verify the mutation of DNA in less than 10 min with a simple visual detection by color change (Figure 2A) [26]. In another work, allele-specific PCR was used in simple colorimetric SNP detection using thiolated primers and an Au-np assay (Figure 2B) [27]. This method was based on the fact that PCR products resulting from perfectly matched thiolated primers inhibit the red-to-blue color transition associated with salt-induced aggregation of Au-nps. At the same time, Au-np solution that does not contain a correct PCR product immediately undergoes aggregation in the presence of salt giving a characteristic colorimetric signal (Figure 2B). Use of 5'-thiolated probes was also adopted in a similar manner for simple post-PCR colorimetric detection of SNP associated with antibiotic resistance in Mycobacterium tuberculosis [28]. Authors underline improved sensitivity of the optical detection using allele-specific oligonucleotides conjugated to Au-nps at lower cost and the possibility to use their assay via a novel portable platform rendering the assay suitable for in-field screening [28]. Finally, a combination of Au-nps with magnetic beads was recently demonstrated to further improve sensitivity of SNP detection in cancer-related KRAS oncogene reaching low picomolar range [29]. In this assay, DNA products to be analyzed were obtained by isothermal amplification (i.e., a PCR-free technique) which utilizes a DNA helicase and single-stranded DNA binding proteins in order to open a double helix, thus avoiding the need for thermal denaturation of the template [30]. A promising aspect of this work is the use of universal poly-T Au-np probes, making the assay quickly adaptable for analysis of various targets [30].

Chart 1. Chemical structures of DNA, RNA and locked nucleic acid (LNA) nucleotide units.

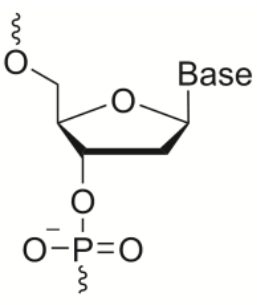

DNA

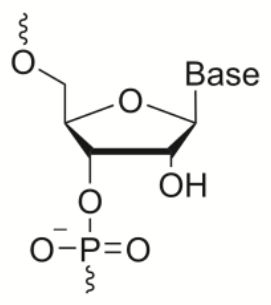

RNA

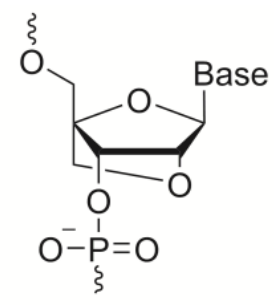

LNA 
Figure 2. Representative detection of SNPs by modified gold nano-particles (Au-nps) using specific probes binding polymerase-chain reaction (PCR) products (A), and allele-specific PCR assay (B) [26,27].

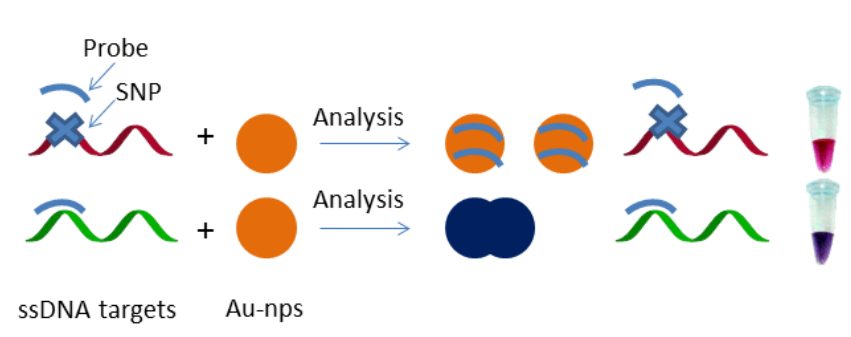

(A)
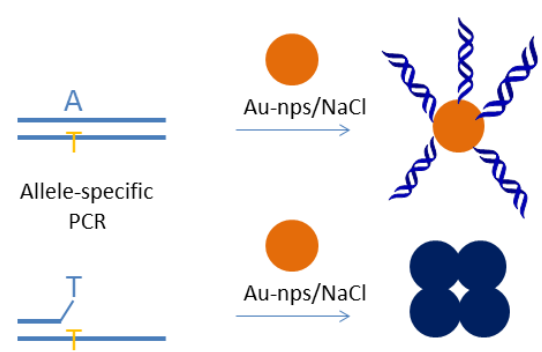

(B)

Another approach to SNP genotyping utilizes single-stranded (ss) oligonucleotides with a stem-and-loop structure called molecular beacons (MBs; Figure 3) [31]. The loop is designed to bind a target sequence, whereas the stem is formed by the hybridization of the arm sequences on either side of the loop sequence. The 5' and 3' termini of MBs are typically labeled with a fluorescent dye and a quencher of its fluorescence. Thus, opening of $\mathrm{MB}$ gives increased fluorescence at a certain wavelength, since the fluorophore is separated from the quencher. As demonstrated by Barreiro and co-authors, an advantage of MBs is the possibility of multiplexing using up to 30 probes to simultaneously interrogate several halotypes in a high-throughput approach [32]. In doing this, SNP detection using $\mathrm{MBs}$ is based on a competition of perfectly-matched and mismatched probes containing different fluorescent dyes for binding to a PCR template. However, disadvantages of MBs in SNP sensing are as follows; a complicated design, false signal due to probe degradation and rather low sensitivity requiring amplification prior to detection [31,32].

Figure 3. Detection of amplified DNA by molecular beacon (MB).
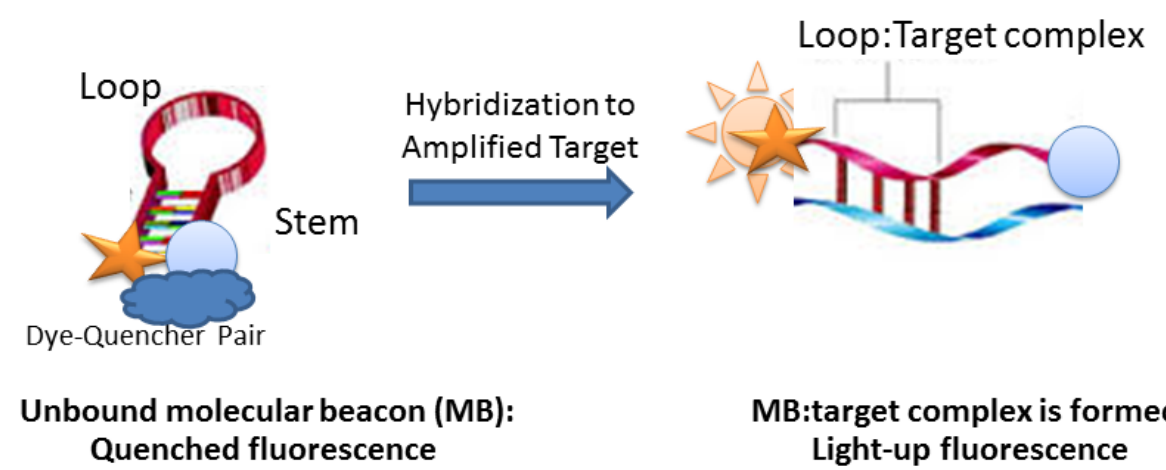

MB:target complex is formed: Light-up fluorescence

Next, visual DNA genotyping can be performed using digital PCR [17] and split DNA enzymes [33]. In the presence of target sequences (i.e., amplified by assymetric PCR) that perfectly matched the complementary probes, the split probes reassembled into active DNAzymes, which catalyzed a colometric reaction. A single-base mismatch prevented this assembly and color change, providing the means for accurate SNP detection with approx. $0.33 \mu \mathrm{M}$ limit of target detection. Alternatively, several enzymatic strategies for SNP detection rely on the high specificity of the enzymes such as thermostable ligases. These enzymes were used in combination with two probes binding adjacent 
regions of the DNA target. Then "ligase chain reaction" of the region containing SNP gave sufficient quantity of ligated probes for detection by Förster resonance energy transfer (FRET) [34]. Another example is the use of flap endonucleases for signal amplification [35]. In these assays, cleavage of the nucleic acid structures occurs when allele-specific probes are displaced by upstream probes at the SNP site of the target. The cleavage results in generation of a fragment which becomes a substrate for secondary reactions, which exponentially generates FRET probes giving detectable signals.

Genotyping by sequencing is another enzyme-based technique for SNP discovery and detection. Among sequencing methods, pyrosequencing has a high sensitivity and represents an ideal method for rapid detection of SNP (e.g., in BRAF gene, a genetic marker of several solid tumors) [36]. The major advantage of sequencing is gaining information on the exact nature of a sequence alteration. Although sequencing is a time-consuming and expensive technique, it is widely used in current SNP diagnostics [37].

Finally, SNP microarrays involve hybridization of the target to a capture probe immobilized on the array surface followed by analysis by diverse methods (incl. PCR and sequence-specific probes such as the aforementioned MBs). Recently developed molecular inversion probe (MIP) assays require as little as $75 \mathrm{ng}$ of genomic DNA for SNP diagnostics on a microarray [38]. Central to the MIP assays are the padlock probes that hybridize to the DNA target of interest before PCR leading to high assay specificity. Four independent nucleotide specific regions are designed for each nucleotide base (A, T, $\mathrm{C}, \mathrm{G})$; sequence-specific thermostable DNA ligase and a cocktail of DNAses to digest unused probes and genomic DNA while leaving the circularized padlock probe intact. The increase in genotyping performance using MIP can be attributed to the dramatic reduction in genome complexity before hybridization to the array. Currently MIP microarrays find multiple applications in cancer genetics [38].

\section{Toward Enzyme-Free Detection of SNP}

As mentioned above, major goals for detection of SNPs in nucleic acids are high specificity and affinity of target binding, bright signal, low cost, high repeatability and robustness of the assays (Figure 1). As illustrated below, several non-enzymatic techniques provide advantages of improved repeatability, decreased cost and robustness. Based on the chemical nature of the utilized probe, these techniques can be divided into three groups: methods applying nano- and magnetic-particles [26-46], (1) aptamer- and protein-based assays [47-52], (2) and other techniques utilizing bright chemically modified nucleic acid analogues [53-60], (3) see Figures 4-5.

\subsection{Nano- and Magnetic-Particles in Amplification-Free SNP Diagnostics}

The complexes between DNA and negatively charged Au-nps mentioned above have been studied for many years [26-29,39], and many creative schemes have exploited covalent complexes of Au-nps with DNA either for nano-assembly or for the specific sensing of nucleic acid targets. Au-np-based colorimetric assays utilize the dispersion and the aggregation of the Au-nps, mainly by cross-linking or non-cross-linking aggregation mechanism [39]. For the crosslinking aggregation mechanisms, several interparticular molecules, such as DNA, antibodies and aptamers, accelerate Au-np aggregation. In turn, the non-cross-linking aggregation mechanism uses changes in electrostatic or (electro)steric stabilization of Au-np surface [40]. Murphy and co-workers applied Au-nps for colorimetric detection 
of SNP in a cystic fibrosis transmembrane conductance regulator (CFTR) gene with sub-picomole sensitivity [41]. In this work, the authors applied a three oligonucleotide system linked to Au-nps wherein two probe oligonucleotide-gold nanoparticle conjugates are linked together by a third target oligonucleotide strand which contains the target mutation in CFTR. Novel miniaturized optical monitoring system and temperature dependence of the optical properties of the complexes allowed to accurately distinguish the mutated nucleotide when present [34]. In another example, allele-specific capture probes were immobilized on microarrays followed by adding a second gene-specific probe after binding the target DNA to the microarray [42]. The second probe contained a nanoparticle which if bound was then visualized on an array by an optical detection system. Remarkably, this enzyme-free, allele-specific assay provided rapid and accurate genotyping of the desired gene fragment using as little as $500 \mathrm{ng}$ total genomic DNA; an amount obtained from a single drop of blood or from a buccal swab.

Next, one microliter of the solutions containing the sub-picomole p53 gene DNA samples was sufficient for microscopy based detection using colored nanospheres [43]. The oligonucleotides were immobilized on organic nanospheres impregnated with fluorescent dyes. Upon the addition of the single-stranded DNA that is complementary to the immobilized probes, the spheres gathered to produce aggregates by cross-linking through specific base-pairing. The colors of the aggregates depended on the added DNA sequences and were monitored using an ordinary fluorescent microscope. FRET between the nanospheres also provided information about the SNP in added DNAs.

Using synthetic nanoparticles prepared by click chemistry between azido groups of oligo ethylene glycol mecrylate (OEGMA) polymer on the surface of silica-coated magnetic nanoparticles with a gene-specific probe, the DNA of herpes simplex (HSV) virus was detected without amplification at low picomolar range (i.e., $6 \mathrm{pM}$ in aqueous buffer and $60 \mathrm{pM}$ in fetal bovine serum) by means of fluorescence (Figure 4A) [44]. The reported methodology of preparation allows control over both OEGMA polymer "brush" thickness and immobilized probe density. After 20 min hybridization with HSV target and the second pool of nanoparticles containing specific HSV probes and fluorescent dye, a wash step and analysis were subsequently performed. Authors mentioned that further development of a particle-counting enzyme-free methodology will result in the development of diagnostic assays with significant improvements in sensitivity [44].

Interestingly, Song and co-workers developed colorimetric SNP detection using intrinsic peroxidase-like activity of single-walled carbon nanotubes (SWNTs; Figure 4B) [45]. In the presence of $\mathrm{H}_{2} \mathrm{O}_{2}$, SWNTs catalyze the reaction of the peroxidase substrate 3,3,5,5,-tetramethylbenzidine (TMB) giving the blue oxidized TMB product (oxTMB). Flexible ssDNA noncovalently interacts with SWNTs with much higher affinity than rigid double-stranded DNA due to active $\pi$-stacking interactions of nucleobases and the side walls of the tube, as well as hydrophobic and van der Waals interactions of ssDNA with SWNTs. Moreover, ssDNA or double-stranded DNA (dsDNA) containing a mismatch inhibit salt-induced aggregation of SWNTs, whereas SWNTs in solution with fully complementary dsDNA are easily precipitated, e.g., in the presence of $0.3 \mathrm{M} \mathrm{Na}^{+}$. The precipitate can be collected, re-dispersed in a buffer and further analyzed by a color reaction in the presence of TMB and $\mathrm{H}_{2} \mathrm{O}_{2}$. Using this enzyme-free assay, SNP has been detected in just $15 \mathrm{~min}$ and at $1 \mathrm{nM}$ concentration of DNA [45]. 
Figure 4. Representative optical methods for enzyme-free detection of SNPs: Oligo ethylene glycol mecrylate (OEGMA)-coated magnetic nanoparticle detecting herpes simplex (HSV) target [44] (A); single-walled carbon nanotube (SWNT) intrinsic peroxidase-like activity in presence of fully complementary and single-mismatched DNA duplex [45]; double-stranded DNA (dsDNA) containing a mismatch inhibit salt-induced aggregation of SWNTs, whereas SWNTs in solution with fully complementary dsDNA are easily precipitated in presence of salt. The precipitate can be collected, re-dispersed in a buffer and analyzed by a color reaction in the presence of 3,3,5,5,-tetramethylbenzidine (TMB) and $\mathrm{H}_{2} \mathrm{O}_{2}(\mathbf{B})$; a colorimetric detection assay using bivalent thrombin-binding aptamers (TBA) and fibrinogen-conjugated Au-nps to detect DNA based on controlled thrombin activity [50] (C).

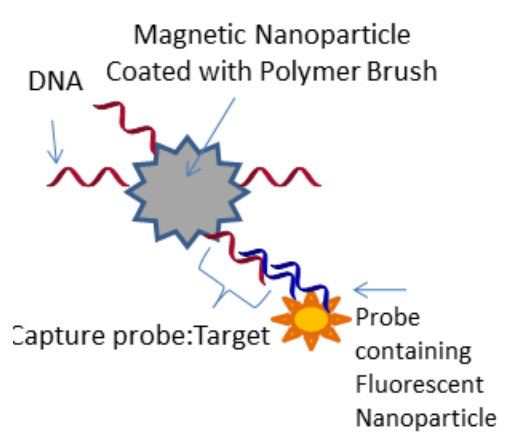

(A)

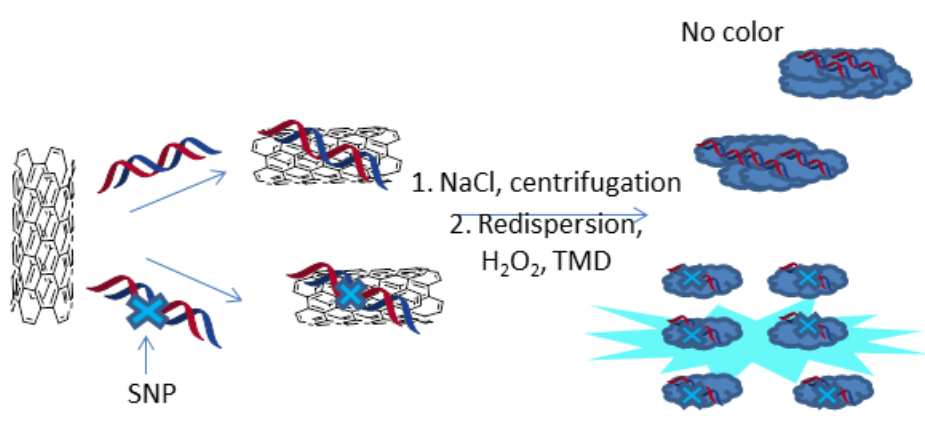

Colorimetric detection of SNP

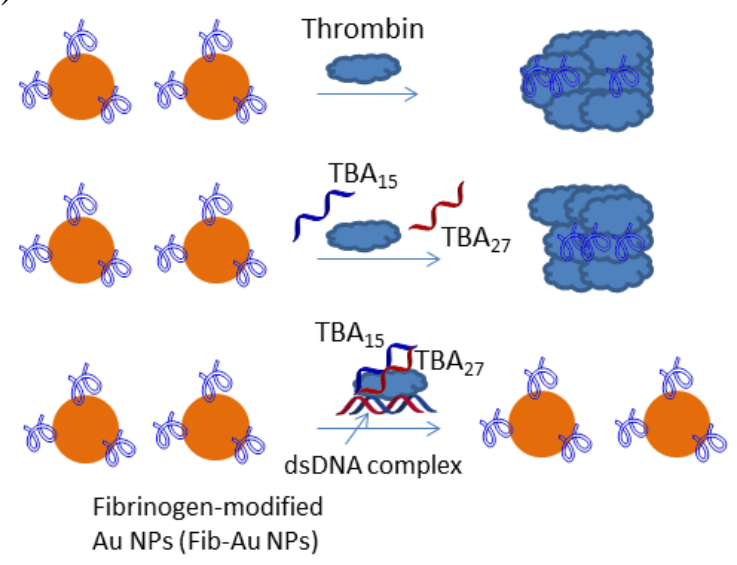

(B)

(C)

Finally, the same sensitivity as PCR ( $70 \mathrm{aM})$ was recently demonstrated for magnetic particles modified with capture DNA sequences in a so-called Simoa assay, which uses biotinylated detection sequences and streptavidin- $\beta$-galactosidase [46]. Remarkably, the Simoa technique is a direct diagnostic system and allows rapid multiplexed detection of nucleic acids and proteins from biologic fluids like blood in a fully automated regimen.

\subsection{Nucleic Acid Aptamers and Proteins in SNP Diagnostics}

Nucleic acid aptamers represent an emerging class of biomolecules for therapeutic and diagnostic applications. DNA aptamers are ss deoxyribonucleic acids which are capable of binding with strong 
affinity and high selectivity to a wide variety of target molecules, such as small organic molecules, carbohydrates and proteins [47]. A colorimetric detection assay was reported using cross-linking [48] or non-cross-linking of Au-nps mediated by DNA aptamers [49]. Among other DNA aptamers, a 29-nt long thrombin-binding aptamer (TBA) has been well studied and already applied in SNP diagnostics [50,51]. Chen and co-workers used self-assembled bivalent thrombin-binding aptamers and fibrinogen-conjugated Au-nps to detect DNA, based on controlled thrombin activity (Figure 4C) [50]. Using this assay, SNP could be detected at $20 \mathrm{pM}$ concentration of target DNA with advantage of confirmed high selectivity. Alternatively, Au-nps modified with 29-nt long thrombin-binding aptamer inhibiting enzymatic activity of thrombin were applied in a sandwich hybridization assay [51]. A second pool of Au-nps modified with thrombin substrate fibrinogen was not converted into aggregated fibrin-Au-nps due to binding of fully complementary DNA to the former Au-nps. This assay allowed detection of DNA by the naked eye at $12 \mathrm{pM}$ concentration, although no information of the type of mismatch could be gained by this method [51]. Finally, a product of an SNP containing gene can also be applied for SNP sensing [52]. This was demonstrated for BRAF oncogene, which in the case of mutation was translated in a distinguished mutant protein serving as a biomarker of SNP.

\subsection{Other Optical Methods for Sensing SNP}

Synthetic nucleotide analogues represent a promising class of sensors which can simultaneously provide increased selectivity and sensitivity in SNP sensing. The aforementioned LNA molecules can be combined with fluorescent dyes in the fluorogenic probe, or the dyes can be directly attached to the 2'-amino position of 2'-amino-LNA (Chart 1; Figure 5) [53-55]. Short fluorescent LNA/DNA probes containing ultrasensitive polyaromatic hydrocarbons (PAHs) such as pyrene [53], (phenylethynyl)pyrenes [54] and perylene [55] represent a class of sensors with advantages of high specificity and sensitivity of sensing SNP. Umemoto and co-workers applied pyrene excimer-forming short LNA/DNA probes for SNP sensing of a model system [53]. We further developed this system and applied an excimer based design for the genotyping of HIV-1 protease (HIV PR) encoding gene fragment (Figure 5) [53]. Using (phenylethynyl)pyrene dyes having improved photophysical characteristics compared to the parent pyrene, the detection limit was below $5 \mathrm{nM}$. Effective detection of SNP was observed also in the presence of one-two polymorphic mutations located 5-7 nucleotides apart from the position of drug-resistance causing SNP (Figure 5B). This system was furthermore efficiently applied for genotyping of clinical samples amplified by PCR. The resulting data was in full agreement with sequencing experiments performed as controls. Finally, FRET was also useful for nucleotide-specific genotyping of the HIV PR gene using LNA/DNA probes [55]. We believe that extraordinary high specificity of LNA/DNA probes and bright fluorescence response to a particular mismatched nucleotide can become a platform for development of the next-generation of enzyme-free SNP diagnostics.

DNAzymes (also called deoxyribozymes or catalytic DNAs) have been recently applied in SNP sensing. Advantages of DNAzymes over traditional protein enzymes include high chemical stability, low cost, simple preparation and easy modification [56]. Tang and co-workers prepared a three-way junction DNAzyme linked to allele-specific hairpin DNA in the presence of the target. DNAzyme in the presence of hemin and fully complementary DNA target forms a G-quadruplex complex catalyzing 
oxidation of 2,2'-azinobis (3-ethylbenzthiazoline-6-sulfonic acid) (ABTS) by $\mathrm{H}_{2} \mathrm{O}_{2}$ to the colored ABTSradical [57]. Using this assay detection was performed at a DNA concentration as low as $0.25 \mathrm{nmol} \cdot \mathrm{L}^{-1}$. In turn, Kolpashnikov described a binary allele-specific peroxidize-like DNA enzyme in SNP diagnostics [58]. Visual fluorimetric analysis using the binary probe revealed SNP in the coding sequence for microtubule associated protein tau (MAPT) at $1 \mu \mathrm{M}$ concentration in $30 \mathrm{~min}$. Promising allosteric nucleic acid sensor molecules based on DNAzymes were reported by Blatt and co-workers [59]. Using this novel design of the multicomponent DNAzyme-based sensor system, authors were able to detect various viral, bacterial, cellular DNA and RNA.

Figure 5. Polyaromatic hydrocarbon (PAH)-LNA/DNA probes in colorimetric assays for SNP detection [54]. HIV-pol = HIV-1 gene fragment encoding protease. (a) Chemical structures and design rules for the PAH-LNA/DNA probes, LNAs are marked with uppercase L; (b) Fluorescence spectra of single-stranded probe and its complexes with complementary (mutant) and mismatched (wild-type) DNA targets.

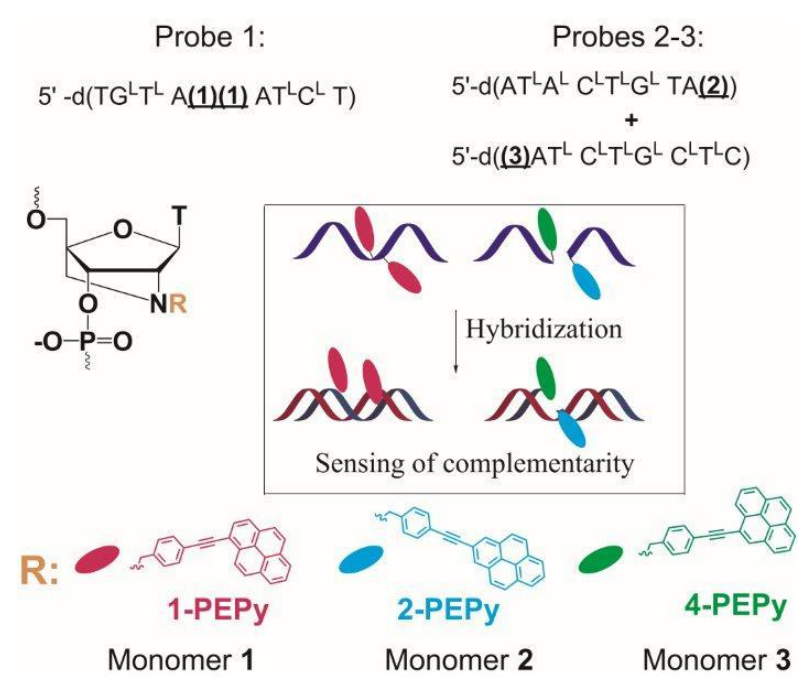

(A)

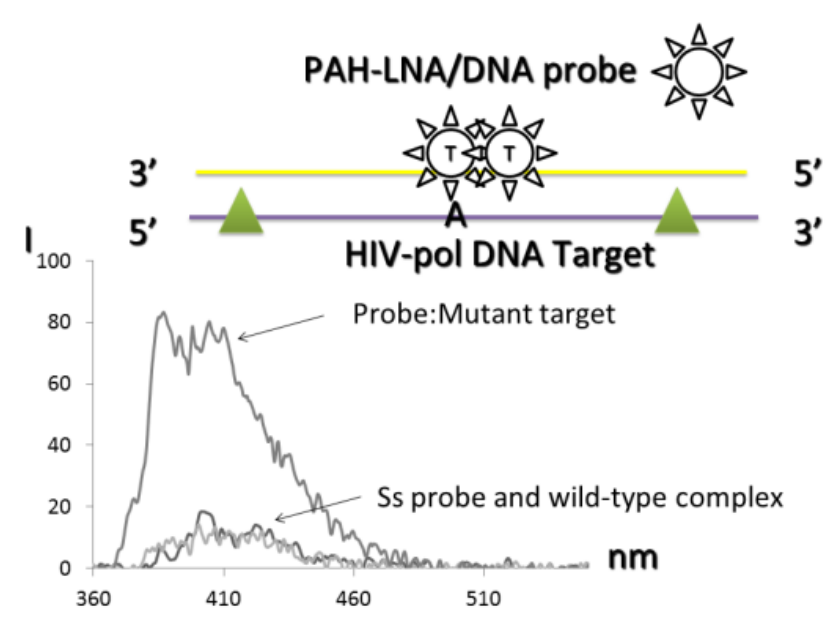

(B)

Finally. exchange of luminescent lanthanide ions $\left(\mathrm{Ln}^{3+}: \mathrm{Tb}^{3+}\right.$ or $\left.\mathrm{Eu}^{3+}\right)$ attached to allele-specific capture probes was demonstrated to be a useful tool in colorimetric SNP sensing of G/C mismatch in the thiopurine S-methyltransferase (TPMT) gene [60]. In doing this, 7mer capture probes, Cwt7 and Cmut7, complementary to wild-type and the mutant part of the target gene, respectively, and carrying EDTA at the 5' terminus, were prepared. The probes Cwt7 and Cmut7 were treated with $\mathrm{Tb}^{3+}$ or $\mathrm{Eu}^{3+}$ prior to SNP detection, respectively. The $20 \mathrm{~m}$ sensor probe containing 1,10-phenanthroline (phen) residue at the 3' terminus was complementary to the rest of both targets. Rapid diagnostics of SNP was based on the characteristic color of the phen complexes of the corresponding ions, although authors do not report a detection limit for their assay.

\section{Conclusions}

To date, multiple strategies have been developed for SNP sensing in nucleic acid targets. Generally, these strategies can be classified as enzyme-based and enzyme-free. Both groups of methods are based on specific binding of the target DNA and its sensing by a method of choice. Although enzymatic 
strategies such as PCR, sequencing and SNP microarrays are well-established in research and clinical laboratories, they are very time consuming and expensive. Moreover, SNP diagnostic methods which rely on protein enzymes often lack specificity and reproducibility. These disadvantages can be solved by the development of non-enzymatic rapid SNP sensing methods. As described in this review, the novel promising SNP probe designs include Au-nps and other biopolymers, DNAzymes, modified nucleic acid analogues, including LNA and PAH-LNA, and complexes of lanthanide ions with nucleic acids. Although most of amplification-free assays still are less sensitive than classic enzyme-based methods, they already have a great advantage of high specificity, low cost and a robust detection technique, often by a simple visually observed change of color. Further development of fluorogenic ultra-bright probes for enzyme-free SNP sensing might benefit from new chemical substances and biotechnological approaches. Herein, sensor probes could be based on recently introduced super-bright bioorthogonal BODIPY-tetrazine dyes [61], new intercalator dyes such as Eva Green [62], nanomaterials modified with PAH-LNA/DNA probes [63] and lanthanide ions [64].

\section{Acknowledgments}

The author would like to acknowledge financial support from The Sapere Aude Programme of The Danish Council for Independent Research (Grant No. FNU 11-115447) and Augustinus Foundation (Project no. 95305 73949).

\section{Conflicts of Interest}

The author declares no conflict of interest.

\section{References and Notes}

1. Sachidanandam, R.; Weissman, D.; Schmidt, S.C.; Kakol, J.M.; Stein, L.D.; Marth, G.; Sherry, S.; Mullikin, J.C.; Mortimore, B.J.; Willey, D.L.; et al. A map of human genome sequence variation containing 1.42 million single nucleotide polymorphisms. Nature 2001, 409, 928-933.

2. Venter, J.C.; Adams, M.D.; Myers, E.W.; Li, P.W.; Mural, R.J.; Sutton, G.G.; Smith, H.O.; Yandell, M.; Evans, C.A.; Holt, R.A.; et al. The sequence of the human genome. Science 2001, 291, 1304-1351.

3. Hacia, J.G. Resequencing and mutational analysis using oligonucleotide microarrays. Nat. Genet. 1999, 21, 42-47.

4. Syvanen, A.-C. Accessing genetic variation: Genotyping single nucleotide polymorphisms. Nat. Rev. Genet. 2001, 2, 930-942.

5. Kwok, P.Y. Methods for genotyping single nucleotide polymorphisms. Annu. Rev. Genomics Hum. Genet. 2001, 2, 235-258.

6. Kirk, B.W.; Feinsod, M.; Favis, R.; Kliman, R.M.; Barany, F. Single nucleotide polymorphism seeking long term association with complex disease. Nucleic Acids Res. 2002, 30, 3295-3311.

7. Koshkin, A.; Rajwanshi, V.K.; Wengel, J. Novel Convenient Syntheses of LNA [2.2.1] Bicyclo Nucleosides. Tetrahedron Lett. 1998, 39, 4381-4384. 
8. Hari, Y.; Obika, S.; Sasaki, M. Effective synthesis of C-nucleosides with 2'-4'-BNA modification. Tetrahedron 2002, 58, 3051-3063.

9. Latorra, D.; Arar, K.; Hurley, J.M. Design considerations and effects of LNA in PCR primers. Mol. Cell. Probes 2003, 17, 253-259.

10. Di Giusto, D.A.; King, G.C. Strong positional preference in the interaction of LNA oligonucleotides with DNA polymerase and proofreading exonuclease activities: Implications for genotying assays. Nucleic Acids Res. 2004, 32, e32.

11. Levin, J.D.; Fiala, D.; Samala, M.F.; Kahn, J.D.; Peterson, R.J. Position-dependent effects of locked nucleic acid (LNA) on DNA sequencing and PCR primers. Nucleic Acids Res. 2006, 34, e142.

12. Rupp, J.; Solbach, W.; Gieffers, J. Single-nucleotide-polymorphism-specific PCR for quantifcation and discrimination of Chlamydia pneumoniae genotypes by use of a "locked" nucleic acid. Appl. Environ. Microbiol. 2006, 72, 3785-3787.

13. Johnson, M.P.; Haupt, L.M.; Griffiths, L.R. Locked nucleic acid (LNA) single nucleotide polymorphism (SNP) genotype analysis and validation using real-time PCR. Nucleic Acids Res. 2004, 32, e55.

14. You, Y.; Moreira, B.; Behlke, M.A.; Owczarzy, R. Design of LNA probes that improve mismatch discrimination. Nucleic Acids Res. 2006, 34, e60.

15. Mouritzen, P.; Nielsen, A.T.; Pfundheller, H.M.; Choleva, Y.; Kongsbak, L.; Møller, S. Single nucleotide polymorphism genotyping using locked nucleic acid (LNATM). Expert Rev. Mol. Diagn. 2003, 3, 27-38.

16. Crowley, E.; Di Nicolantonio, F.; Loupaki, F.; Bardelli, A. Liquid biopsy: Monitoring cancer-genetics in the blood. Nat. Rev. Clin. Oncol. 2013, 10, 472-484.

17. Didelot, A.; Kotsopoulos, S.K.; Lupo, A.; Pekin, D.; Li, X.; Atochin, I.; Srinivasan, P.; Zhong, Q.; Olson, J.; Link, D.R.; et al. Multiplex Picoliter-Droplet Digital PCR for Quantitative Assessment of DNA Integrity in Clinical Samples. Clin. Chem. 2013, 59, 815-823.

18. Østergaard, M.E.; Hrdlicka, P.J. Pyrene-functionalized oligonucleotides and locked nucleic acids (LNAs): Tools for fundamental research, diagnostics, and nanotechnology. Chem. Soc. Rev. 2011, 40, 5771-5788.

19. Xiao, Y.; Lou, X.; Uzawa, T.; Plakos, K.J.I.; Plaxco, K.W.; Soh, H.T. An Electrochemical Sensor for Single Nucleotide Polymorphism Detection in Serum Based on a Triple-Stem DNA Probe. J. Am. Chem. Soc. 2009, 131, 15311-15316.

20. Cagnin, S.; Caraballo, M.; Guiducci, C.; Martini, P.; Ross, M.; Santaana, M.; Danley, D.; West, T.; Lanfranchi, G.; et al. Overview of Electrochemical DNA Biosensors: New Approaches to Detect the Expression of Life. Sensors 2009, 9, 3122-3148.

21. Huang, T.J.; Liu, M.; Knight, L.D.; Grody, W.W.; Miller, J.F.; Ho, C.-M. An electrochemical detection scheme for identification of single nucleotide polymorphisms using hairpin-forming probes. Nucleic Acids Res. 2002, 30, e55.

22. Zhang, J.; Wu, X.Y.; Yang, W.; Chen, J.; Fu, F.F. Ultra-sensitive electrochemical detection of single nucleotide polymorphisms based on an electrically controllable magnetic gold electrode. Chem. Commun. 2013, 49, 996-998. 
23. Guo, Y.; Chen, J.; Chen, G. A label-free electrochemical biosensor for detection of HIV related gene based on interaction between DNA and protein. Sens. Actuators B. Chem. 2013, 184, 113-117.

24. Wei, F.; Lillehoj, P.B.; Ho, C.-M. DNA Diagnostics: Nanotechnology-Enhanced Electrochemical Detection of Nucleic Acids. Pediatr. Res. 2010, 67, 458-468.

25. Astakhova, I.K.; Kumar, T.S.; Campbell, M.A.; Ustinov, A.V.; Korshun, V.A.; Wengel, J. Branched DNA nanostructures efficiently stabilised and monitored by novel pyrene-perylene 2'- $\alpha$-L-amino-LNA FRET pairs. Chem. Commun. 2013, 49, 511-513.

26. Li, H.; Rothberg, L.J. Label-Free Colorimetric Detection of Specific Sequences in Genomic DNA Amplified by the Polymerase Chain Reaction. J. Am. Chem. Soc. 2004, 126, 10958-10961.

27. Jung, Y.-L.; Jung, C.; Parab, H.; Cho, D.Y.; Park, H.G. Colorimetric SNP genotyping based on allele-specific PCR by using a thiol-labeled primer. ChemBioChem 2011, 12, 1387-1390.

28. Veigas, B.; Machado, D.; Perdigão, J.; Portugal, I.; Couto, I.; Viveiros, M.; Baptista, P.V. Au-nanoprobes for detection of SNPs associated with antibiotic resistance in Mycobacterium tuberculosis. Nanotechnology 2010, 21, 415101-415108.

29. Valentini, P.; Fiammengo, R.; Sabella, S.; Gariboldi, M.; Maiorano, G.; Cingolani, R.; Pompa, P.P. Gold-nanoparticle-based colorimetric discrimination of cancer-related point mutations with picomolar sensitivity. ACS Nano 2013, 7, 5530-5538.

30. Vicent, M.; Xu, Y.; Kong, H. Helicase-dependent isothermal DNA amplification. EMBO Rep. 2004, 5, 795-800.

31. Marras, S.A.; Kramer, F.R.; Tyagi, S. Genotyping SNPs with Molecular Beacons. Methods Mol. Biol. 2002, 212, 111-128.

32. Barreiro, L.B.; Henriques, R.; Mhlanga, M.M. High-throughput SNP genotyping: Combining tag SNPs and molecular beacons. Methods Mol. Biol. 2009, 578, 255-276.

33. Neo, J.L.; Uttamchandani, M. Visual SNP genotyping using asymmetric PCR and split DNA enzymes. Analyst 2011, 136, 1569-1572.

34. Wabuyele, M.B.; Farquar, H.; Stryjewski, W.; Hammer, R.P.; Soper, S.; Cheng, Y.W.; Barany, F. Approaching real-time molecular diagnostics: Single-pair fluorescence resonance energy transfer (spFRET) detection for the analysis of low abundant point mutations in K-ras oncogenes. J. Am. Chem. Soc. 2003, 125, 6937-6945.

35. Hall, J.G.; Eis, P.S.; Law, S.M.; Reynaldo, L.P.; Prudent, J.R.; Marshall, D.J.; Allawi, H.T.; Mast, A.L.; Dahlberg, J.E.; Kwiatkowski, R.W.; et al. Sensitive detection of dna polymorphisms by the serial invasive signal amplification reaction. Proc. Natl. Acad. Sci. USA 2000, 97, 8272-8277.

36. Spittle, C.; Ward, M.R.; Nathanson, K.L.; Gimotty, P.A.; Rappaport, E.; Brose, M.S.; Medina, A.; Letrero, R.; Herlyn, M.; Edwards, R.H.; et al. Application of a BRAF Pyrosequencing Assay for Mutation Detection and Copy Number Analysis in Malignant Melanoma. J. Mol. Diagn. 2007, 9, 464-471.

37. Gessi, M.; Pietsch, T. The Diagnostic Role and Clinical Relevance of Determination of BRAF Status in Brain Tumors. Pers. Med. 2013, 10, 405-412. 
38. Wang, Y.; Cottman, M.; Schiffman, J.D. Molecular inversion probes: A novel microarray technology and its application in cancer research. Cancer Genet. 2012, 205, 341-355, and references cited therein.

39. Mirkin, C.A.; Letsinger, R.L.; Mucic, R.C.; Storhoff, J.J. A DNA-Based Method for Rationally Assembling Nanoparticles into Macroscopic Materials. Nature 1996, 382, 607-609.

40. Kang, J.-H.; Asami, Y.; Murata, M.; Kitazaki, H.; Sadanaga, N.; Tokunaga, E.; Shiotani, S.; Okada, S.; Maehara, Y.; Niidome, T.; et al. Gold nanoparticle-based colorimetric assay for cancer diagnosis. Biosens. Bioelectron. 2010, 25, 1869-1874.

41. Murphy, D.; O'Brien, P.; Redmond, G. Sub-picomole colorimetric single nucleotide polymorphism discrimination using oligonucleotide-nanoparticle conjugates. Analyst 2004, 129, 970-974.

42. Bao, Y.P.; Huber, M.; Wei, T.F.; Marla, S.S.; Storhoff, J.J.; Müller, U.R. SNP identification in unamplified human genomic DNA with gold nanoparticle probes. Nucleic Acids Res. 2005, 33, e15.

43. Ihara, T.; Tanaka, S.; Chikaura, Y.; Jyo, A. Preparation of DNA-modified nanoparticles and preliminary study for colorimetric SNP analysis using their selective aggregations. Nucleic Acids Res. 2004, 32, e105.

44. Thomson, D.A.C.; Tee, E.H.-L.; Tran, N.T.D.; Monteiro, M.J.; Cooper, M.A. Oligonucleotide and polymer functionalized nanoparticles for amplification-free detection of DNA. Biomacromolecules 2012, 13, 1981-1989.

45. Song, Y.; Wang, X.; Zhao, C.; Qu, K.; Ren, J.; Qu, X. Label-Free Colorimetric Detection of Single Nucleotide Polymorphism by Using Single-Walled Carbon Nanotube Intrinsic Peroxidase-Like Activity. Chem. Eur. J. 2010, 16, 3617-3621.

46. ELISA-inspired technology developed in so-called Simoa instrument by Quanterix, founded 2007 by D. Walt and N. Naclerio.

47. Gold, L.; Polisky, B.; Uhlenbeck, O.; Yarus, M. Diversity of Oligonucleotide Functions. Annu. Rev. Biochem. 1995, 64, 763-797.

48. Huang, C.-C.; Huang, Y.F.; Cao, Z.; Tan, W.; Chang, H.T. Aptamer-modified gold nanoparticles for colorimetric determination of platelet-derived growth factors and their receptors. Anal. Chem. 2005, 77, 5735-5741.

49. Zhao, W.; Chiuman, W.; Brook, M.A.; Li, Y. Simple and rapid colorimetric biosensors based on DNA aptamer and noncrosslinking gold nanoparticle aggregation. ChemBioChem 2007, 8, 727-731.

50. Chen, C.-K.; Shiang, Y.-C.; Huang, C.-C.; Chang, H.-T. Using self-assembled aptamers and fibrinogen-conjugated gold nanoparticles to detect DNA based on controlled thrombin activity. Biosens. Bioelectron. 2011, 26, 3464-3468.

51. Jian, J.-W.; Huang, C.-C. Colorimetric Detection of DNA by Modulation of Thrombin Activity on Gold Nanoparticles. Chem. Eur. J. 2011, 17, 2374-2380.

52. Capper, D.; Berghoff, A.-S.; von Deimling, A.; Preusser, M. Clinical neuropathology practice news 2-2012: BRAF V600E testing. Clin. Neuropathol. 2012, 31, 64-66. 
53. Umemoto, T.; Hrdlicka, P.; Babu, B.R.; Wengel, J. Sensitive SNP Dual-Probe Assays Based on Pyrene-Functionalized 2'-Amino-LNA: Lessons to Be Learned. ChemBioChem 2007, 8, 2240-2248.

54. Astakhova, I.K.; Samokhina, E.; Babu, B.R.; Wengel, J. Novel (Phenylethynyl)pyrene-LNA Constructs for Fluorescence SNP Sensing in Polymorphic Nucleic Acid Targets. ChemBioChem 2012, 13, 1509-1519.

55. Kumar, T.S.; Myznikova, A.; Samokhina, E.; Astakhova, I.K. Rapid genotyping using pyrene-perylene locked nucleic acid complexes. Artif. XNA DNA PNA 2013, 4, 58-68.

56. Breaker, R.R. DNA enzymes. Nat. Biotechnol. 1997, 15, 427-431.

57. Tang, S.; Tong, P.; Li, H.; Gu, F.; Zhang, L. The three-way junction DNAzyme based probe for label-free colorimetric detection of DNA. Biosens. Bioelectron. 2013, 41, 397-402.

58. Kolpashnikov, D.M. Split DNA Enzyme for Visual Single Nucleotide Polymorphism Typing. J. Am. Chem. Soc. 2008, 130, 2934-2935.

59. Blatt, L.; Chowrira, B.; Haeberli, P.; Jadhav, V.; Kossen, K.; Mcswiggen, J.; Seiwert, S.; Usman, N.; Vaish, N.; Zinnen, S. Allosteric nucleic acid sensor molecules. WO2003089650 A2, 2003.

60. Kitamura, Y.; Ihara, T.; Tsujimura, Y.; Osawa, Y.; Jyo, A. Colorimetric allele analysis based on the DNA-directed cooperative formation of luminous lanthanide complexes. Nucleic Acids Symp. Ser. 2006, 50, 105-106.

61. Carlson, J.C.T.; Meimetis, L.G.; Hilderbrand, S.A.; Weissleder, R. BODIPY-Tetrazine Derivatives as Superbright Bioorthogonal Turn-on Probes. Angew. Chem. Int. Ed. 2013, 52, 6917-6920.

62. Mao, F.; Leung, W.-Y.; Xin, X. Characterization of EvaGreen and the implication of its physicochemical properties for qPCR applications. BMC Biotechnol. 2007, 7, 76.

63. Astakhova, I.K.; Wengel, J. Scaffolding along Nucleic Acid Duplexes Using 2'-Amino-Locked Nucleic Acids. Acc. Chem. Res. 2014, 47, 1768-1777.

64. Wirpsza, L.; Pillai, S.; Batish, M.; Marras, S.; Krasnoperov, L.; Mustaev, A. Highly Bright Avidin-based Affinity Probes Carrying Multiple Lanthanide Chelates. J. Photochem. Photobiol. B. 2012, 116, 22-29.

(C) 2014 by the authors; licensee MDPI, Basel, Switzerland. This article is an open access article distributed under the terms and conditions of the Creative Commons Attribution license (http://creativecommons.org/licenses/by/3.0/). 